\title{
A floristic Description of the Afromontane fynbos communities on Platberg, Eastern Free State, South Africa
}

\author{
ROBERT F. BRAND \\ Department of Plant Sciences, \\ University of the Free State \\ South Africa \\ PIETER J. DU PREEZ \\ Department of Plant Sciences \\ University of the Free State \\ South Africa \\ LESLIE R. BROWN \\ Applied Behavioural Ecology and Ecosystem Research Unit \\ Department of Environmental Sciences \\ University of South Africa \\ South Africa \\ Correspondence to: Pieter J. du Preez \\ e-mail: dpreezpj.sci@ufs.ac.za \\ Postal Address: Department of Plant Sciences, UFS, PO Box 339, Bloemfontein
}

\begin{abstract}
Within the Platberg area and the wider Drakensberg region, the shrinking natural resources and the threat posed to biodiversity are of concern to conservation management and require an understanding of long-term ecological processes. The vegetation of Platberg was investigated as part of an ecological survey to establish Afromontane floristic links to the Drakensberg as well as for the management of natural resources. From a TWINSPAN classification, refined by the Braun-Blanquet method, four main plant communities were identified, which were subdivided into fynbos, wetland, a woody/ shrub community and grassland. A classification and description of the fynbos are presented in this article.

The analysis showed the fynbos divided into two communities comprising four sub-communities and seven variants. The fynbos community had an average of 28.34 species per relevé, ranging from 14 to 54 species per sample plot. Twenty-four endemic or near-endemic Drakensberg Alpine Centre (DAC) species and 22 exotic (alien-invasive) species were recorded. Numerous floristic links with the DAC Cape flora fynbos and grassland bioregions to the north and west were also found. The description of the fynbos plant communities can serve as a basis for the formulation of management plans for the area.
\end{abstract}

Keywords: Free State province, Drakensberg Alpine Centre (DAC) of endemism, Braun-Blanquet method, Afromontane fynbos communities, Platberg

Indigenous biodiversity is under increasing pressure from numerous forces that threaten a loss of species and the depaupering of natural resources. Platberg, in the Eastern Free State, overlooking the town of Harrismith and is an island surrounded by plains of grassland. This inselberg represents a refuge for indigenous Afromontane plants and animals.

Prior to this study, no extensive vegetation surveys had been undertaken on Platberg. Limited opportunistic floristic collections had been done in the mid-1960s by Mrs ML Jacobs, and some 50 relevés had been sampled by Prof. HJT Venter of the University of the Free State (UFS) between 1975 and 1977 (vouchers from these collectors are housed at the Geo Potts herbarium (BLFU) of the UFS).

Due to the proposed commercial development of parts of the Platberg conservancy as well as the aims of the Maluti Drakensberg transfrontier project, it was therefore considered necessary to undertake a more detailed and extensive ecological survey to describe and classify the vegetation of Platberg. These data will be used by the Free State Provincial Department of Tourism, Economic and Environmental Affairs as part of its biological-diversity survey and conservation-management strategies. The data may also be incorporated into the Maluti
Drakensberg transfrontier-project vegetation maps for future land use and conservation management.

\section{STUDY AREA}

The town of Harrismith is located at the foot of Platberg (latitude $\mathrm{S} 29^{\circ} 10^{\prime}$ and longitude E $28^{\circ} 16^{\prime}$ ) on the N3 highway between Johannesburg and Durban (Fig. 1). Platberg's altitude ranges from $1900 \mathrm{~m}$ to $2394 \mathrm{~m}$; its surface area covers approximately 3000 ha. The slopes are steep, with numerous vegetated gullies and boulder-scree slopes below the vertical cliffs, which are 20 to $45 \mathrm{~m}$ high. Waterfalls cascade down the southern cliffs after rain. A permanent stream arising from the manmade dam (Gibson Dam) flows off the escarpment, cascading into a waterfall.

Woody patches of the genera Leucosidea, Buddleja, Kiggelaria, Polygala, Heteromorpha and Rhus shrubs as well as the only indigenous mountain bamboo, Thamnocalamus tessellatus, grow along the bases of the cliffs, on sandstone of the Clarens Formation, in the gullies, on the scree slopes, in the mobile boulder beds, on the rocky ridges and as riparian vegetation in the cleft, in which the only road ascends steeply to the summit. Locally, the pass is aptly named Donkey Pass for its steepness 


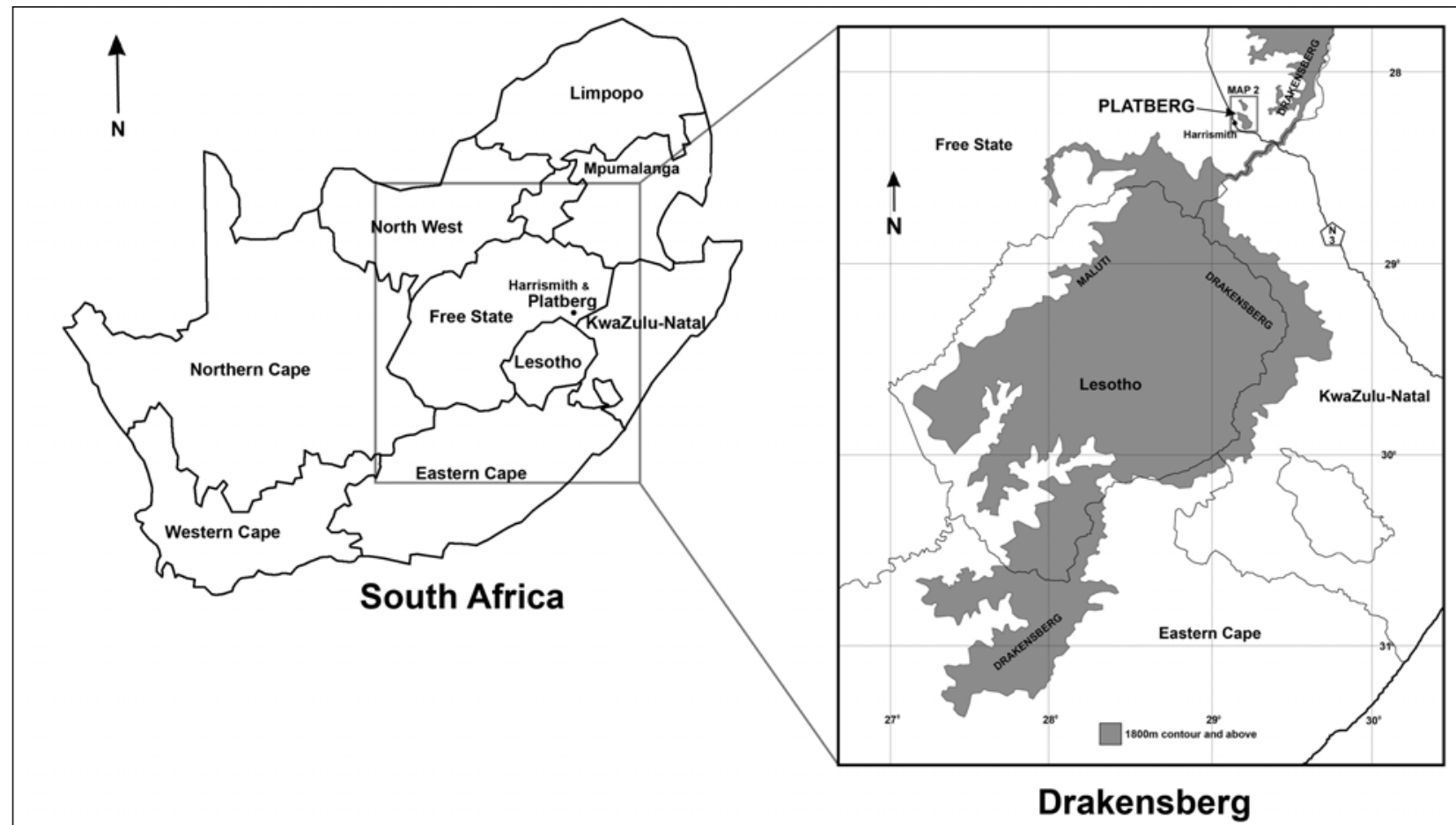

MAP 1

FIGURE 1
Map of region

and sheer sides. It bypasses a waterfall that drains the Gibson Dam to the east. From a distance, Platberg appears to have a distinct flat top; once on the summit plateau, however, it is obvious that it has undulating, rolling, grass-covered plains instead. The vegetation of the plateau is dominated by grassland - with a few rocky ridges, sheet rock and rubble patches - by numerous seasonal wetlands and by a few permanent open playas (pans) on the far-western side of the Platberg plateau.

Acocks $(1952,1988)$ classifies the vegetation of the Drakensberg region into the following veld types: Highveld sourveld (44a), Themeda-Festuca alpine veld (58), Stormberg Plateau sweetveld (59) and sclerophyllous bush types, and fynbos (69). As a result of the 1:1 500000 scale of Acocks's map (1988), however, Platberg does not appear as a distinct vegetation unit and is therefore included in the larger grassland biome.

Low and Rebelo (1996) give a more detailed description of the vegetation of the region, indicating the following vegetation types: wet, cold Highveld grassland (41), moist upland grassland (42), Afro mountain grassland (45) and alti mountain grassland (46).

Platberg falls within the grassland biome, which generally contains short to tall sour grasses (Acocks, 1988; Low and Rebelo, 1996). Mucina and Rutherford (2006) designate the study area as part of the Drakensberg grassland bioregion. It also has elements of fynbos, false karroo (Acocks, 1988) or succulent Karoo (Low and Rebelo, 1996) as well as elements of temperate and transitional forest, specifically 44a Highland sourveld (Acocks, 1952, 1988) veld types.

The most recent and detailed vegetation description presented by Mucina and Rutherford (2006) shows that the study area falls within the Eastern Free State Sandy Grassland (Gm 4), which is part of the broader Mesic Highveld Grassland bioregion. The Platberg plateau is shown as an island that belongs to the Drakensberg Grassland bioregion. Embedded within this bioregion are a series of isolated patches of fynbos, designated by Mucina and Rutherford (2006) as Drakensberg-Amathole Afromontane fynbos (Gd6). Mucina and Rutherford (2006) additionally recognise two structurally similar but floristically different Afromontane fynbos shrublands in the Drakensberg and surrounding area. A species of poor, fynbos-like shrubland with Passerina montana is found on the edges of some of the high Eastern Free State inselbergs (Mucina \& Rutherford, 2006). Floristically, fynbos-like shrubland elements found on Platberg show strong affinities with Passerina montana, a sub-unit of the Gd 8 fynbos-type vegetation, which, according to Mucina and Rutherford (2006) still has hundreds of patches to be mapped.

\section{Geology}

Platberg consists of layers of the Karoo supergroup, which, stratagraphically, lies immediately below the volcanics of the Drakensberg formation, the youngest unit of the Stormberg group. The $1900 \mathrm{~m}$ contour forms the start to the footslopes of Platberg and is constituted by the aeolian and water-borne sediments of the Clarens formation (Du Toit, 1954; Loock, Praekelt, Van der Westhuizen \& Welman, 1991; Norman \& Whitfield, 1998; Truswell, 1970). Several dolerite dykes intersect Platberg, with a close-set sequence of parallel dykes forming the woody/shrub community on the northern and southern slopes. A dolerite dyke is evident just below the Gibson Dam, shown in a borrow pit used for quarrying. Numerous other dykes are visible on aerial photographs. These dyke swarms occur mostly on the flat terrain at lower altitudes, with only a few intersecting Platberg itself. Some of the dykes are also visible in plantation road-cuts on the southern side of Platberg.

\section{Topography and soils}

Fig. 2 shows a terrain-form sketch of the study area. The steep slopes are composed of mudstone and mostly sandstone of the Clarens formation, intersected by dolerite dykes, which results in benches, deep ravines and terraces. Numerous sandstone and basalt boulders form scree and boulder slopes, resulting 


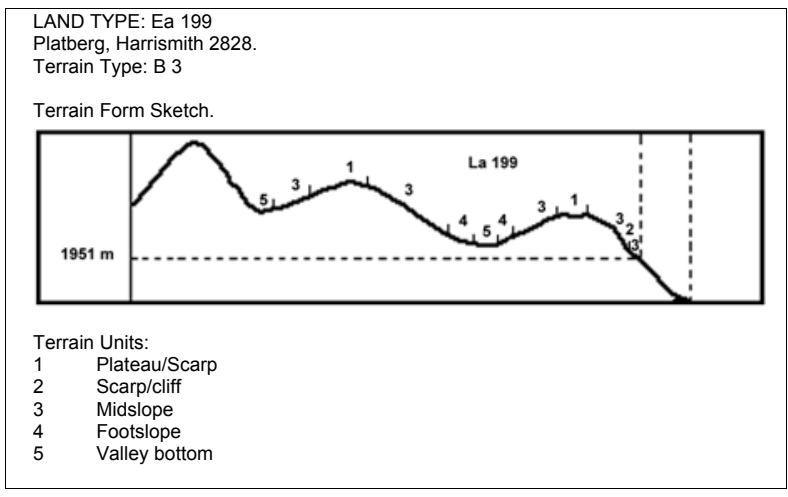

FIGURE 2

Landscape sketch showing the principal terrain units. (after Land Types Survey Staff 1991)

in miscellaneous soils forms characteristic of the Ib land type, specifically Mispah (Ms) and Glenrosa (Gs) soil forms (Soil classification working group 1991 Du Preez, 1991; Mucina and Rutherford, 2006). There are affinities with Fa land types, undulating rocky landscape dominated by shallow Ms, and Gs soil forms (Land Type Survey Staff, 1991; Smit, Bredenkamp and Van Rooyen, 1992)

The summit plateau is composed of igneous rock. The soils are generally shallow (20 to $200 \mathrm{~mm}$ ), with the deepest being $<500 \mathrm{~mm}$. They are derived from basalt and are shown to have even proportions of course sand, fine sand, clay, silt and moderate to high organic-matter content. The main land type is Ea (clay vertic, melanic or red diagnostic horizons) (Erickson 1985, Soil classification working group 1991; Mucina and Rutherford, 2006; Smit et al., 1992; Taylor, 1996), which has a clay content of 8 to $60 \%$, depending on the soil series (Land Type Survey Staff, 1991).

\section{Climate}

The average annual precipitation for the study area ranges between 800 and $1200 \mathrm{~mm}$ per annum. Daily mean maximum temperatures fluctuate between 22 and $24^{\circ} \mathrm{C}$ for the hottest month of January; during the coldest months (June and July), the daily mean temperature ranges from minus 2 to $0^{\circ} \mathrm{C}$, respectively. The daily mean relative humidity for the most humid month of March varies between 68 and $72 \%$ and the daily minimum relative humidity for July and August between 32 and 38\% (Schulze, 1997; Van Zinderen Bakker, 1973).

Snow is recorded to cover the top of Platberg during some winters and can stay on the ground for days. Water in southfacing gullies may be frozen for several weeks at a time (Van Zinderen Bakker, 1973). Possible heave soils are present on Mtabazwe, the highest point on Platberg (Hilliard and Burtt, 1987), and may be significant factors affecting vegetation and structure at high altitudes (Hilliard and Burtt, 1987).

The climate of Platberg and the Drakensberg is part of a much longer, continental, north-south trending austro-afroalpine climate, with noticeably higher precipitation than the surrounding lowland. Precipitation along the Drakensberg escarpment is over $1400 \mathrm{~mm}$ in some areas, with a gradual decrease both to the north and the south as well as westwards $(560 \mathrm{~mm})$ to the drier, sub-humid, continental-climate interior towards Bloemfontein, some $400 \mathrm{~km}$ away (Van Zinderen Bakker, 1973; Scott, 1988). Precipitation is orographic, not only from violent thunderstorms in summer but also from dense mists and fogs (Smit et al., 1992) as well as snow. Climatic heterogeneity associated with montane landscapes is, for Cowling, Holmes and Rebelo (1992), a more importan determinant of plant diversity than geological heterogeneity.

\section{Inselbergs: Their importance and uniqueness}

Plant communities that occur on inselbergs form unique phytosociological associations (Porembski and Brown, 1995; Parmentier, Oumorou, Porembski, Lejoly \& Decocq, 2006; Sarthou and Villiers, 1998). However, inselberg plant associations also have numerous species that occur in the vegetation matrix surrounding the inselbergs (Mutke, Kier, Braun, Schultz and Barhlott, 2001) and in which the inselberg flora is embedded (Platberg and Korannaberg fall within the grassland biome).

The unique, high-altitude conditions found above $2000 \mathrm{~m}$ lead to high levels of endemism in organisms, plants, animals and bryophytes (Carbutt and Edwards, 2006; Haore and Bredenkamp, 2001; Hilliard and Burtt, 1987; Mucina and Rutherford, 2006; Smit et al., 1992; Van Wyk and Smith, 2001). This is due to the compression of climatic life zones over a short distance that makes mountains hot spots for biological diversity (Körner, 2003). Mountains may be regarded as analogous with an archipelago of islands in an "ocean" of low-level vegetation types that act as an isolation factor (MacArthur and Wilson, 2001; Taylor, 1996). This, in turn, precludes plant species with less mobile seed-dispersal mechanisms from propagating over wide ranges and allows for high levels of endemism to develop (Hilliard and Burtt, 1987; Taylor, 1996). High levels of endemism mean that a large proportion of the available gene pool is unique to that site, and inselbergs and mountains therefore have an important role to play in the maintenance of genetic diversity (Mucina and Rutherford, 2006; Taylor, 1996).

\section{METHODS}

Platberg was chosen as the study site as it is the largest inselberg situated approximately $60 \mathrm{~km}$ north of the main massif of the Maluti/Drakensberg range (Chief Directorate: Survey and Mapping, 2000). It is also the highest peak in the area, at $2394,5 \mathrm{~m}$ (survey beacon 44), as well as the largest inselberg capped by igneous rock (Du Toit, 1954; Erickson, 1985; King, 1963; Norman and Whitfield, 1998). It forms a distinctive flattopped, L-shaped plateau, with clear-cut contact between the Stormberg volcanics and the cave sandstone of the Clarens formation, clearly visible from afar.

The analysis of 38 relevés, which were extracted from a total of 393 relevés for the entire Platberg, was used to constitute the fynbos community on Platberg. The scope of the study was to sample above the $1800 \mathrm{~m}$ contour in order to work within the limits set by Killick (1978), who regarded the region in the Drakensberg above $1800 \mathrm{~m}$ as a distinct floristic region: the Afroalpine region. The topography of the region is relatively flat and rises abruptly at the $1900 \mathrm{~m}$ contour - this being the start of the footslopes of Platberg - to the highest point of $2394 \mathrm{~m}$.

Plot sizes were fixed at $6 \times 5 \mathrm{~m}$ to give a total surface area of $30 \mathrm{~m}^{2}$ (Du Preez, 1991; Malan, 1998; Whittaker, 1980). In all the sample plots, each species was recorded, all the woody plants were counted and all the cover/abundance was calculated with the Braun-Blanquet cover-abundance scale (Behr and Bredenkamp, 1988; Brown, Marais, Henzi and Barrett, 2005; Clever, Brown and Bredenkamp, 2005; Mueller-Dombois and Ellenberg, 1974; Westhoff and Van der Maarel, 1980; Whittaker, 1980). Ad hoc floristic collections were undertaken in 2004, with the main fieldwork starting in February/March 2005, and continued during the following summers in the growing season from October to December 2005 through to the end of March 2006. Final follow-up fieldwork was done in January 2007.

Over 1500 floristic collections were made and identified at either the Geo Potts herbarium (BLFU) or the national herbarium (PRE) in Pretoria. Germishuizen and Meyer (2003) were used 
to confirm the names of the taxa. The specimens are currently housed at the Geo Potts herbarium of the UFS.

Environmental data recorded included aspect, slope, exposure, the size of the rocks, latitude and longitude, altitude, locality, geology, the per cent of area covered by rock, topography, the degree of surface erosion, the degree of trampling, drainage, soil depth and notes on management and utilisation as well as total percentage canopy cover.

Numerous phytosociological studies (Barrett, Brown, Barrett and Henzi, 2006; Bredenkamp and Brown, 2003; Du Preez, 1991, 1992; Eckhardt et al., 1995; Eckhardt, Van Rooyen and Bredenkamp, 1993; Grobler, Bredenkamp and Brown, 2002; Hoare and Bredenkamp, 2001; Malan, 1998; Pond, Beesley, Brown and Bezuidenhout, 2002; Smit, Bredenkamp and Van Rooyen, 1995) have shown that the Braun-Blanquet classification (Kent and Coker, 1992; Whittaker, 1980) is the most efficient phytosociological method to use. Habitat as well as floristic data were processed with TURBOVEG (Hennekens, 1996a). A first approximation was done with the TWINSPAN (two-way indicator-species analysis) algorithm of Hill (1979a). MEGATAB (Hennekens, 1996b) was used to generate a phytosociological table, which was refined by Braun-Blanquet procedures. A DECORANA (Hill, 1979b) analysis was also applied to the floristic data to determine the relationship between the various vegetation units and the environmental gradients.

Erosion was estimated with the three-scale numerical system, where $1=$ no erosion, $2=$ moderate and $3=$ high. Slope was estimated in the following scale in degrees: 0 to $3^{\circ}=$ flat, 3 to $8^{\circ}=$ gradual, 8 to $16^{\circ}=$ moderate, 16 to $26^{\circ}=$ steep, 26 to $45^{\circ}=$ very steep, and greater than $45^{\circ}$. Soil depths were measured with a probe graded for $5 \mathrm{~cm}$ intervals to a maximum of $30 \mathrm{~cm}$.

Hand-held Garmin Magellan 2X and Etrex global positioningsystem instruments were used to give latitude, longitude, aspect and altitude for the sample plots. Altitudes were also read from 1:50 000 topographical maps.

\section{RESULTS}

A total of 299 species (Table 1) was recorded in 38 sample plots. The fynbos community has an average of 28.34 species per relevé, ranging from 14 to 54 species per sample plot. The fynbos is dominated by several low, sclerophyllous shrubs approximately $1 \mathrm{~m}$ high on the rocky outcrops, summit plateau, vertical cliffs, steep scree slopes and cave sandstone of the Clarens formation. A mixture of low shrubs, grasses, forbs and succulents characterises the fynbos community for the study site.

\section{Vegetation classification}

The analysis resulted in the identification of two communities, four sub-communities and seven variants. The phytosociological classification is presented in Table 1 and is as follows:

1. Tristachya leucothrix--Eragrostis curvula grassland community

1.1 Eragrostis curvula--Themeda triandra grassland subcommunity

2.2 Eragrostis curvula--Eragrostis racemosa grassland subcommunity

2.2.1 Passerina montana variant

2.2.2 Harpochloa falx variant

2. Helichrysum dasycephalum--Digitaria monodactyla grassland community

1.1 Bulbostylis humilis--Passerina montana subcommunity

1.1.1 Watsonia lepida variant

1.1.2 Cliffortia ramosissima variant
1.1.3 Ischyrolepis schoenoides variant
1.1.4 Eragrostis capensis variant
Cliffortia nitidula variant
sarcocaulis--Cliffortia
2.2 Crassula
nitidula
subcommunity

\section{Description of plant communities}

The study area is mostly open grassland, with scattered fynbos communities either on rocky outcrops, on the summit plateau area or concentrated on the steep slopes and cliffs of Platberg on the sandstone of the Clarens formation. Little disturbance from grazing is evident, with no record of agricultural practice on the plateau of Platberg. There are some clusters and scattered individuals of the declared alien invasive tree species Pinus patula (species group E), having escaped from plantations established at lower altitudes and now being firmly ensconced on the footslopes and scattered on the summit plateau area.

The sclerophyllous vegetation is characterised by Passerina montana (species group M, Table 1), Cliffortia ramosissima (species group F, Table 1) and Cliffortia nitidula (species group P, Table 1). Common species throughout the study area include the forbs Hebenstretia dura, Watsonia lepida, Commelina africana, Oxalis obliquifolia, Senecio glaberrimus, Sutera floribunda and Polygala amatymbica, the xerophytic fern Cheilanthes quadripinnata, the perennial tufted grasses Eragrostis curvula and Melinis nerviglumis, the two sedges Carex zuluensis and Ficinia stolonifera, the succulent Crassula lanceolata subsp. transvaalensis and the geophyte Merwilla plumbea (species group P, Table 1). The geophyte Agapanthus campanulatus and the climber Cyphia elata (species group O, Table 1) occur in all the communities.

\section{Tristachya leucothrix-Eragrostis curvula grassland community}

The Tristachya leucothrix-Eragrostis curvula grassland is located on flat to moderately steep rocky terrain on the ridge lines and steep slopes. Rock cover varies between 10 and 40\%, with sandy soil between 40 and $250 \mathrm{~mm}$ deep. On the steep slopes, high erosion is present, with no erosion occurring on the flat areas.

This community is characterised by the single-bunch grass Tristachya leucothrix (species group A, Table 1).

Vegetation is dominated by the perennial grass Eragrostis curvula (species group P), the low fynbos shrub Passerina montana (species group M) and the dwarf shrub Selago galpinii (species group F). Other species of importance include the perennial grass Tristachya leucothrix (species group A), the restio Ischyrolepis schoenoides (species group G), Eragrostis curvula (species group P), the forb Watsonia lepida and the sedge Ficinia stolonifera (species group P, Table 1).

The total species count is 338, which gives an average of 30.7 species per $30 \mathrm{~m}^{2}$.

There are two sub-communities and two variants:

1.1 Eragrostis curvula--Themeda triandra grassland subcommunity

The Eragrostis curvula-Themeda triandra sub-community is located on the northern and north-western slopes, with gradients varying between 8 and $26^{\circ}$, in full sun and in soil depths varying between 40 and $60 \mathrm{~mm}$. The vegetation occurs on the contact between the cave sandstone of the Clarens formation and the igneous cap at approximately $2200 \mathrm{~m}$. Surface erosion is high, with rock cover estimated at $15 \%$. The area is overgrazed, with concomitant high levels of erosion due to impact by cattle.

Species belonging to species group B, Table 1, are diagnostic for this community and include the geophyte Chlorophytum cooperi, the forbs Cyanotis speciosa and Acalypha punctata and the perennial grass Themeda triandra. 
TABLE 1

Fynbos Communities of Platberg, Eastern Free State

\begin{tabular}{|c|c|c|c|c|c|c|c|c|c|c|c|c|c|c|c|c|c|c|c|c|c|c|c|c|c|c|c|c|c|c|c|}
\hline & 2 & & & & 32 & 11 & & & 22 & 23 & 32 & 22 & 23 & & & 23 & & & & 2 & & 22 & & 3 & 2 & 2 & & 1 & 3 & & \\
\hline & 6 & 25 & 56 & & 56 & 83 & 34 & - 4 & 68 & 82 & 27 & 7 & 92 & 2 & 77 & 73 & & 37 & 9 & 9 & 2 & 25 & 5 & 5 & 77 & 72 & 1 & 5 & 3 & 8 & 7 \\
\hline & 4 & 90 & 01 & 0 & 26 & 52 & 25 & 7 & 7 & 9 & 9 & 91 & 18 & 8 & 76 & 63 & & 16 & 4 & 3 & 6 & 32 & 2 & 5 & 32 & 27 & 9 & 5 & 0 & 7 & 9 \\
\hline Database no. & & & & & & & & & & & & & & & & & & & & & & & & & & & & & & & \\
\hline & 3 & 33 & 33 & 3 & 33 & 33 & 33 & 3 & 33 & 33 & 3 & 33 & 33 & 3 & 33 & 33 & & 33 & 3 & 3 & 3 & 33 & 3 & 3 & 33 & 33 & 3 & 3 & 3 & 3 & 3 \\
\hline & 7 & 77 & 77 & 7 & 77 & 77 & 77 & 7 & 77 & 77 & 7 & 77 & 77 & 7 & 77 & 77 & & 77 & 7 & 7 & 7 & 77 & 7 & 7 & 77 & 77 & 7 & 7 & 7 & 7 & 7 \\
\hline & 4 & 22 & 22 & 2 & 54 & 33 & 35 & 5 & 4 & 45 & 5 & 4 & 45 & 5 & 34 & 45 & & 22 & 4 & 4 & 4 & 44 & 4 & 5 & 42 & 24 & 2 & 3 & 5 & 2 & 2 \\
\hline & 6 & 35 & 56 & 3 & 57 & 83 & 34 & 5 & 7 & 93 & 3 & 8 & 93 & 3 & 88 & 83 & & 38 & 9 & 9 & 3 & 25 & & 5 & 77 & 73 & 2 & 5 & 3 & 9 & 8 \\
\hline & 8 & 34 & 45 & 4 & 60 & 96 & 69 & 1 & 1 & 33 & 3 & 35 & 52 & 2 & 10 & $\begin{array}{ll}07 \\
0\end{array}$ & & 50 & 8 & 7 & 0 & 76 & 6 & 9 & 76 & 1 & 3 & 9 & 4 & 1 & 3 \\
\hline Number of species per relevé & 2 & 22 & 22 & 1 & 23 & 42 & 24 & 4 & 33 & 32 & 2 & 25 & 53 & & 22 & 22 & & 13 & 3 & & & 24 & & 2 & 23 & 32 & 3 & 1 & 2 & 1 & 3 \\
\hline & 5 & 72 & 21 & 6 & 81 & 19 & 95 & 0 & $3 \varepsilon$ & 83 & 3 & 84 & 46 & 6 & 53 & 36 & & 71 & 2 & 0 & 0 & 26 & 6 & 9 & 72 & 2 & 6 & 7 & 6 & 3 & 5 \\
\hline Community & & & & & 1 & & & & & & & & & & & & & & & & 2 & & & & & & & & & & \\
\hline Sub-community & & 1. & .1 & & & 1.2 & & & & & & & & & & & & 2.1 & & & & & & & & & & & & 2.2 & \\
\hline Variant & & & & & 1.2 .1 & & 1.2 .2 & & & & 2.1 & & & & & .1 .2 & & & 1.3 & & & 2.1 .4 & & & 2.1 . & 1.5 & & & & & \\
\hline Species group $\mathrm{A}$ & & & & & & & & & & & & & & & & & & & & & & & & & & & & & & & \\
\hline Tristachya leucothrix & + & & & & $\mathrm{b}$ & & a & & $\cdot$ & $\cdot+$ & + & . . & .. & . & .. & . & & · & $\cdot$ & $\cdot$ & . & .. & . & . & . & . & . & $\cdot$ & . & + & \\
\hline Species group B & & & & & & & & & & & & & & & & & & & & & & & & & & & & & & & \\
\hline Themeda triandra & & $b$. & . . & & . & . . & . . & & . & . & . & . . & . . & . & . & . . & & . . & . & . & & + & & . & . . & . & & . & . & . & . \\
\hline Cyanotis speciosa & $\cdot$ & ++ & ++ &. & $r$ & . . & . . & & . & . & . & . . & . . & . & . & . . & & + . & . & . & . & . . & . & . & . . & . & . & . & . & . & . \\
\hline Chlorophytum cooperi & . & + . & . . & $r$ & . & . . & . . & & . & . & . & . . & . . & . & . & . . & & + . & . & . & . & . . & . & . & . . & . & . & . & . & . & . \\
\hline Acalypha punctata & + & & . & + & . & . . & . . & . & $\cdot$ & $\cdot$ & . & . & . . & $\cdot$ & . & . . & & . . & . & $\cdot$ & . & . . & . & · & $\cdot$. & . & . & . & . & . & . \\
\hline Species group C & & & & & & & & & & & & & & & & & & & & & & & & & & & & & & & \\
\hline Eragrostis racemosa & . & . . & . . & . & 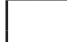 & a $a$ & $a b$ & & $\cdot$ & . & . & . . & . . & . & . . & . . & & . $b$ & . & . & . & . . & . & . & . . & . & . & . & . & . & \\
\hline Heteropogon contortus & & . . & . . & . & L. & b b & $b \quad a$ & $\mathrm{~b}$ & $\cdot$ & $\cdot$ & . & .. & $\cdot$ & & . & . & & + & + & $\cdot$ & . & . . & . & . & $\cdot$. & . & . & . & . & . & . \\
\hline Species group D & & & & & & & & & & & & & & & & & & & & & & & & & & & & & & & \\
\hline Harpochloa falx & . & . . & . . & . & + & b $b$ & $b+$ & & . & $\cdot$ & . & . . & .. & . & . & . . & & . . & + & . & . & . . & . & . & . . & & . & . & · & + & \\
\hline Helichrysum nudifolium & . & + . & . . & . & · $\cdot$ & + . & . + & + & . & . & . & . . & . . & . & . & . . & & . . & . & . & . & . . & . & . & . . & - . & . & . & . & . & - \\
\hline Wahlenbergia depressa & . & . . & . . & . & . . & . . & . $r$ & $r$ & . & . & . & . . & . . & . & . & . . & & . . & . & . & . & . . & . & . & . . & . & . & . & . & . & - \\
\hline Senecio paucicalyculatus & . & . . & . . & . & . & . $r$ & $r \quad r$ & & $\cdot$ & . & . & . . & . . & . & . & . . & & . . & $\cdot$ & . & . & . . & . & . & . . & . & . & . & . & . & . \\
\hline Species group $\mathrm{E}$ & & & & & & & & & & & & & & & & & & & & & & & & & & & & & & & \\
\hline Aristida monticola & . & . . & . . & & . & $\mathrm{b}$. & . & & &.$a^{2}$ & $a$. & . . & . b & & . & . . & & . . & . & . & . & . . & . & . & . . & . & . & . & + & . & a \\
\hline Cymbopogon dieterlenii & . & . . & . . & & . . & . & . & . & . & . & & . & & & . & . . & & . . & . & . & . & . . & . & . & . . & . & . & . & $\cdot$ & . & . \\
\hline Pinus pinaster & . & . . & . $b$ & . & . . & $r$ & . . & . & . & . & . & . . & . + & & . & . . & & . . & . & . & . & . . & . & . & . . & . & . & . & . & . & - \\
\hline Eragrostis chloromelas & 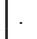 & . . & . . & . & . & . . & . & . & . & $\cdot+$ & + & . . & . + & & . & . . & & . . & . & . & . & . . & . & . & . . & .. & . & . & . & . & - \\
\hline Eucomis bicolor & . & . . & . . & + & · $\cdot$ & . . & . . & . & . & $r$ & . & . . & . $r$ & r & . & . . & & . . & . & . & . & . . & . & . & . . & .. & + & . & . & . & . \\
\hline Rhus dentata & 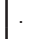 & . + & + . & . & . . & . . & . . & . & $\cdot$ & $\cdot$ & & . . & . . & . & . & . . & & . . & . & . & . & . . & . & . & . . & . & . & . & $\cdot$ & . & . \\
\hline Clutia affinis & + & . . & . . & . & . & . . & . + & . & . & $\cdot+$ & + & . . & . . & & . & . . & & . . & . & . & . & . . & . & . & . . & . & . & . & . & . & . \\
\hline Rhus discolor & $a$ & & & $\dot{-}$ & & & & + & + & & & 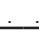 & & & . & . & & . & . & . & . & . . & 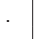 & . & . . & . & . & . & . & . & . \\
\hline Species group $\mathrm{F}$ & & & & & & & & & & & & & & & & & & & & & & & & & & & & & & & \\
\hline Selago galpinii & & ++ & ++ & . & ++ & 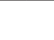 & 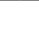 & + & + & ++ & + & . + & + & & & ++ & & . . & . & . & . & . . & . & . & . . & . & . & . & + & . & . \\
\hline Cliffortia ramosissima &. & . & . & . & . b & . . & . . & . & & . & & . . & . & & & a & & . . & . & $b$ & . & . $a$ & a & . & . . & -. & . & . & . & . & . \\
\hline Senecio subrubriflorus & & . & - & $\dot{-1}$ & & & + & & & + & & 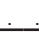 & & & & + & & . & . & . & . & . . & . & . & . . & - . & . & . & . & . & . \\
\hline Species group G & & & & & & & & & & & & & & & & & & & & & & & & & & & & & & & \\
\hline Ischyrolepis schoenoides & . & . & . . & . & & & . $a$ & & . & 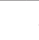 & . & . & . & & & . . & & $b$ & $\mathrm{~b}$ & $\mathrm{~b}$ & . & . . & . & . & . . & . & . & . & $\cdot$ & . & - \\
\hline Crassula peploides & . & . + & + . & . & . & . + & + & . & $\cdot+$ & + & . & . . & . . & & & + . & & $+r$ & + & $\mathrm{r}$ & . & . . & . & . &.+ & + & . & . & . & . & . \\
\hline Macowania glandulosa & & . . & . . & . & $\mathrm{b}$ & . . & . . & . & $\cdot$ & . & $\cdot$ & . . & .. & & . & $\cdot$ & & . . & + & + & . & . + & + & . & . . & . & . & . & · & . & . \\
\hline Aster perfoliatus & & + . & . . & . & $\cdot$ & ++ & + . & . & $\cdot$ & . & . & .. & $\cdot+$ & + & & . $r$ & & . . & . & $\cdot$ & . & . . & . & . & . . & . & . & . & . & . & - \\
\hline Helichrysum sutherlandii & & + . & . & . & . . &. &. & . & . &. &. &.+ & + . & & & + & &. & . & $r$ & . & . . & . & . & . . & . & . & . & . & . & . \\
\hline Species group $\mathrm{H}$ & & & & & & & & & & & & & & & & & & & & & & & & & & & & & & & \\
\hline Digitaria monodactyla & + & . . & . . & . & $\cdot \mathrm{b}$ & . . & . . & . & $a$ & a & 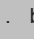 & $\mathrm{b}$ & & & $+b$ & b & & . & a & & b & 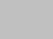 & & . & a. & & . & & & & \\
\hline Helichrysum dasycephalum & . & + . & . . & . & $\cdot+$ & . . & . . & . & + & + & . & + & & & & . + & & b & a & & . & . . & & + & $+b$ & 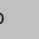 & . & . & . & & \\
\hline Cyperus schlechteri & . & . . & . . & . & . . & . . & . . & . & & + & . & + & + & & + & . & & . . & . & . & . & . + & + & & + . & . & . & & + & & \\
\hline Crassula dependens & . & . . & . . & . & . . & + & . . & . & & . & . + & ++ & ++ & & $r+$ & + & & . . & + & + & . & . . & . & . &.+ & . & . & $\mathrm{b}$ & . & . & . \\
\hline Schizocarphus nervosa & . & . . & . . & . & . . & . . & . . & & & . & . & $\cdot+$ & + . & & ++ & + & &.+ & . & . & + & + . & . & . & + & + & . & + & . & + & + \\
\hline Ruschia putterillii & . & . . & . . & . & . & . . & . . & & & . & & . & & . & . & . & & . . & & . & . & . + & + & . & . . & . & . & & . & . & \\
\hline Psammotropha mucronata & . & . . & . . & . & · $\cdot$ & . . & . + & & & . & $\cdot$ & + & & & $+r$ & r & & ++ & + & & . & . . & . & & $r+$ & $r$ & & & . & + & + \\
\hline Helichrysum ecklonis & . & . . & . . & . & · $\cdot$ & . . & . . & . & rata & . & . & . & & . & .. & . $r$ & & . + & + & + & . & . . & & . & . . & - . & + & . & . & . & + \\
\hline Stoebe vulgaris & . & . . & . . & . & . . & . . & . . & . & &.+ & + & . & & & . . & . $b$ & & . . & & . & . & . & & . & . & . & . & . & + & . & . \\
\hline Dimorphotheca jucunda & . & . . & . & . & . & . . & . . & . & & ++ & + & . & & & . & . + & & $r$ & & & . & . & & & . & . & . & & + & . & \\
\hline Muraltia saxicola & & + . & . . & . & . & . . & . & . & & + & & . & & . & + & & & & & 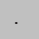 & . & - & & & 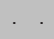 & . & . & 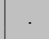 & & . & \\
\hline Lotononis lotononoides & & + . & . . & & . & . . & . . & & & & & . & & & & & & & & & & & & & & & + & & & & \\
\hline Species group I & & & & & & & & & & & & & & & & & & & & & & & & & & & & & & & \\
\hline Bulbostylis humilis & - & . . &. & . & . &. & . + & & + & + & . & ++ & ++ & + & ++ & + & & 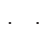 & + & + & a & $+r$ & & . & + & - & & 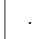 & . & + & \\
\hline Crassula lanceolata & . & . . & . . & . & . . & $r$. & . $r$ & + & . & . & 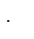 & . & . . & & . $\mathrm{r}$ & $\mathrm{r}$ & & . & $r$ & . & . & $r$ & & . & $\cdot+$ & $r$ & + & . & . & . & . \\
\hline Senecio othonniflorus & . & . . & . . & & . . & . . & . . & + & & + & . & . & . . & . & . & . . & & . $r$ & + & . & . & & . & . &.+ & . & & . & + & . & \\
\hline Cycnium racemosum & . & . . & . . & & . . & . . & . . & & . & $\cdot+$ & + & . & . . & . & . & . & & . . & + & . & . & . + & + & . & + . & . & & . & & . & . \\
\hline Sebaea natalensis & . & . . & . . & · & . & . . & . . & & & + & & +1 & $r$. & & . & . & & + & & . & . & . . & & 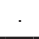 & . & . & & . & & & + \\
\hline
\end{tabular}

Plant species with a presence of 4 or less have been omitted. 
TABLE 1 (CONT...)

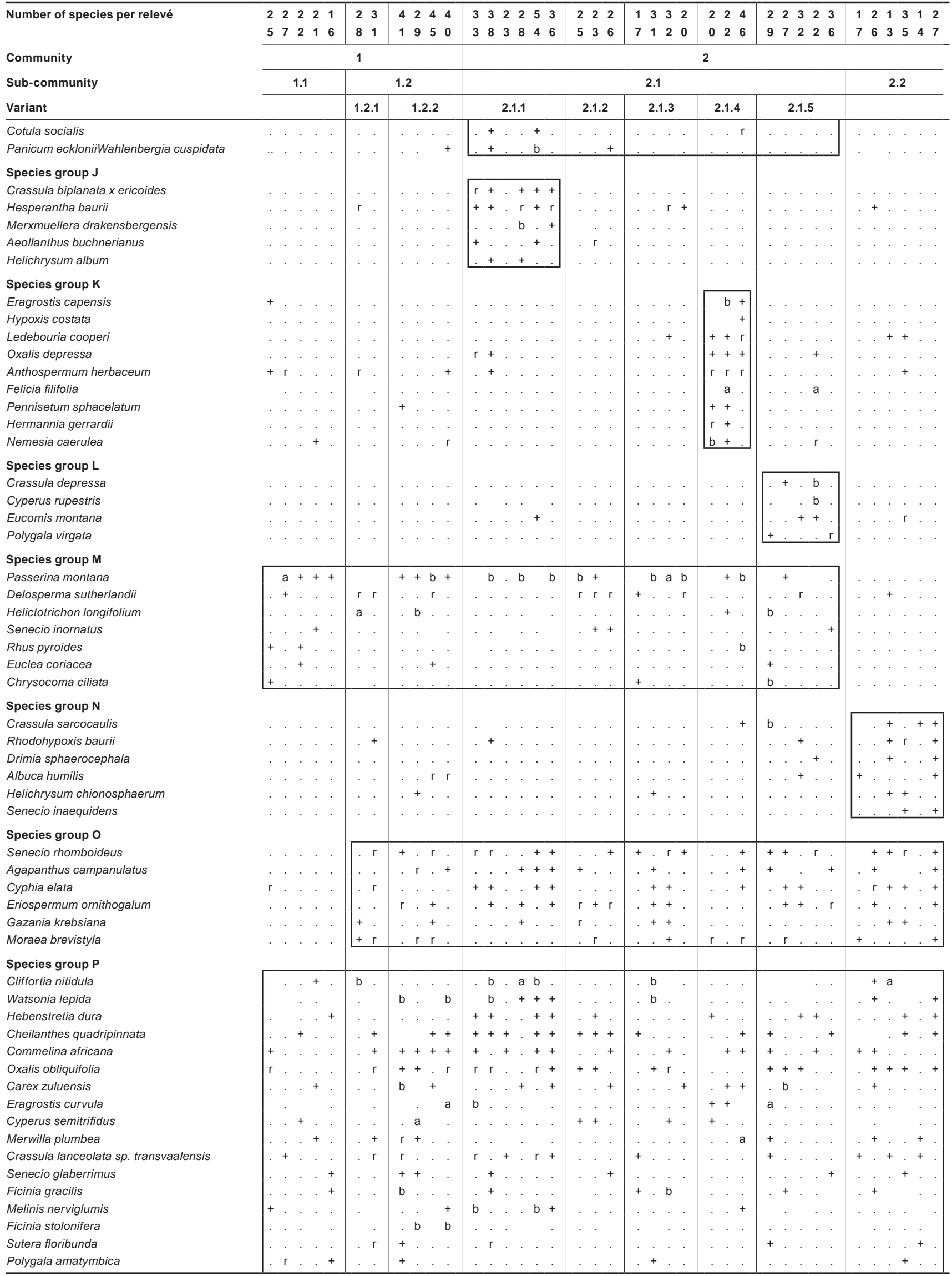

Plant species with a presence of 4 or less have been omitted. 
The vegetation has no dominant species but it does have significant cover abundance by the low shrubs Passerina montana (species group M) and Selago galpinii (species group F), the perennial grasses Themeda triandra (species group B) and Eragrostis curvula (species group P) and the forb Watsonia lepida (species group $\mathrm{P}$ ), with a dense stand of the invader exotic tree Pinus patula (species group E, Table 1).

The total species count is 111, which gives an average of 22.2 species per $30 \mathrm{~m}^{2}$.

\subsection{Eragrostis curvula--Eragrostis racemosa grassland sub- community}

The Eragrostis curvula-Eragrostis racemosa sub-community is located on rocky terrain, on steep to very steep slopes (16 to $45^{\circ}$ ), in the sandstone of the Clarens formation, and on the cool, southern or south-western slopes. The community is in full sun on rocky outcrops of between 10 and $40 \%$. Soil depths are shallow $(100 \mathrm{~mm})$ or $>300 \mathrm{~mm}$ deep, a mix of sandstone and basalt, and well drained.

The short tufted grasses Eragrostis racemosa and Heteropogon contortus (species group C, Table 1) are diagnostic and also the dominant species for this sub-community. The sclerophyllous shrub Passerina montana (species group M, Table 1) is also codominant.

Other species of importance include the grass Tristachya leucothrix (species group A), Aristida monticola (species group E), Eragrostis curvula, (species group $\mathrm{P}$ ), the restio Ischyrolepis schoenoides (species group G), Watsonia lepida, the sedge Ficinia stolonifera (species group P) and the dwarf shrub Selago galpinii (species group F)

The total species count is 222 , which gives an average of 37 species per $30 \mathrm{~m}^{2}$.

\subsubsection{Passerina montana variant}

This variant occurs on the southern aspects, on the steep to very steep $\left(16\right.$ to $\left.45^{\circ}\right)$ mid-slopes, on a series of rocky ledges and semi-vertical cliffs (with 10 to $25 \%$ exposure), and in the cave sandstones of the Clarens formation. Soils are shallow (40 to $100 \mathrm{~mm}$ ) and well drained, and show no trampling or erosion.

The vegetation is dominated by the sclerophyllous shrub Passerina montana (species group M), with the restio Ischyrolepis schoenoides (species group G) and the perennial grass Eragrostis racemosa (species group $\mathrm{C}$ ) being co-dominant. Other species with significant cover abundance include the perennial grasses Tristachya leucothrix (species group A), Heteropogon contortus (species group C) and Helictotrichon longifolium (species group M), and the fynbos shrubs Cliffortia ramosissima (species group F), Macowania glandulosa (species group G) and Cliffortia nitidula (species group P).

The community is depaupered in geophytes, herbs and forbs, with an average species per $30 \mathrm{~m}^{2}$ of 28.1 .

\subsubsection{Harpochloa falx variant}

This variant is located on the mid-slope at $2100 \mathrm{~m}$ on moderate to steep ( 28 to $45^{\circ}$ ) slopes. Rockiness is between 10 and $50 \%$, with dry, shallow soils 40 to $120 \mathrm{~mm}$ deep. No trampling or erosion was seen, despite the steep slope. The grass Harpochloa falx and the forbs Helichrysum nudifolium, Wahlenbergia depressa and Senecio paucicalyculatus (species group D) are diagnostic for this variant.

The vegetation is dominated by the grasses Eragrostis racemosa and Heteropogon contortus (species group C). Important species include the grasses Tristachya leucothrix (species group A),
Harpochloa falx (species group D) and Aristida monticola (species group E), Eragrostis curvula, Melinus nerviglumis, the sedges Carex zuluensis, Cyperus semitrifidus and Ficinia stolonifera (species group P), the restio Ischyrolepis schoenoides (species group G), the shrubs Passerina montana and Selago galpinii (species group F) and the fern Chelianthes quadripinnata (species group P).

The total species count is 165 , which gives an average of 41.2 species per $30 \mathrm{~m}^{2}$.

2. Helichrysum dasycephalum-Digitaria monodactyla grassland community

The Helichrysum dasycephalum-Digitaria monodactyla community is the largest fynbos community on Platberg. It is located on the southern aspects, on the flat, rocky ridge line on the summit plateau and on the steep, exposed mid-slope along the cave sandstone. Soils are well drained and shallow (40 to $180 \mathrm{~mm}$ ) or $>300 \mathrm{~mm}$ deep. Rock cover varies between 10 and 50\%, with little or no erosion or trampling visible.

Species belonging to group $\mathrm{H}$ are diagnostic for this community and include the grass Digitaria monodactyla, the forbs Helichrysum dasycephalum, Psammotropha mucronata, Helichrysum eckloni and Dimorphotheca jucunda, the sedge Cyperus schlecteri, the succulents Crassula dependens and Ruschia putterllii, the geophyte Schisocarpus nervosa, the fynbos dwarf shrub Muraltia saxicola and the shrub Lotononis lotononoides.

The community is dominated by the grass Digitaria monodactyla (species group $\mathrm{H}$ ) and the sclerophyllous shrub Passerina montana (species group M). Important species include the forb Helichrysum dasycephalum, the sedge Cyperus schlecteri (species group $\mathrm{H}$ ), the shrub Cliffortia nitidula, the bulb Watsonia lepida, the sedges and grasses Ficinia gracilis and F. stolonifera, Eragrostis curvula and Melinis nerviglumis (species group P), respectively.

The total species count is 752 , which gives an average of 27.9 species per $30 \mathrm{~m}^{2}$.

\subsection{Bulbostylis humilis-Passerina montana sub-community}

The Bulbostylis humilis--Passerina montana sub-community occurs in full sun on the gently sloping ridge line $\left(8^{\circ}\right)$ on the summit basalt and on the steep slopes $\left(26\right.$ to $45^{\circ}$ ) of the cave sandstone. Rock exposure varies between 15 and 30\%. Little or no erosion, with moderate to no trampling, was noted. Soils are either shallow (40 to $160 \mathrm{~mm}$ ) or $>300 \mathrm{~mm}$ deep, well drained and a mix of weathered basalt, sandstone and dolerite. Canopy cover for the shrubs is $<2 \mathrm{~m}$ high and is 5 to $15 \%$, with the grasses, forbs and herbs forming a low sub-layer.

The species diagnostic for this community include the small sedge Bulbostylis humilis, the small erect succulent Crassula lanceolata, the forb Senecio othonniflorus, the root parasite Cycnium racemosa, Sebaea natalensis, Cotula socialis, Wahlenbergia cuspidata and the grass Panicum ecklonii (species group I).

The vegetation is dominated by the grass Digitaria monodactyla and the forb Helichrysum dasycephalum, with co-dominance of the fynbos shrubs Passerina montana (species group M). Other significant species include Cliffortia nitidula, the bulb Watsonia lepida, the grasses Eragrostis curvula and Melinis nerviglumis, the sedges Ficinia gracilis and F. stolonifera and the geophyte Merwilla plumbea (species group P).

The total species count is 620 , which gives an average of 29.5 species per $30 \mathrm{~m}^{2}$.

\subsubsection{Watsonia lepida variant}

This variant is located in full sun, on the hot western and northern aspects, on the moderate to very steep slopes $\left(16\right.$ to $\left.45^{\circ}\right)$ and on the mid-slope and plateau area. Altitudes vary from 2100 to $2200 \mathrm{~m}$ 
for the slopes and $2300 \mathrm{~m}$ for the plateau. Rock exposure varies between 10 and $25 \%$. No trampling or erosion was noted. Soil depths vary between 20 and $200 \mathrm{~mm}$. The variant was located either on basalt of the Drakensberg volcanics or cave sandstone of the Clarens formation.

The diagnostic species for this variant are the geophyte Hesperantha baurii and the succulent Crassula biplanata x ericoides, with the tall mountain grass Merxmuellera drakensbergensis and the forbs Aeolanthus buchnerianus and Helichrysum album (species group J).

The grass Digitaria monodactyla (species group $\mathrm{H}$ ), together with the fynbos shrub Passerina montana (species group M), dominates this vegetation. Species with significant cover include the shrubs Cliffortia ramosissima (species group F) and Cliffortia nitidula (species group P), the perennial grasses Cymbopogon dieterlenii and Aristida monticola (species group E), the restio Ischyrolepis schoenoides (species group G), the sedge Cyperus schlecteri (species group H), Eragrostis curvula, Melinis nerviglumis, the geophyte Schisocarpus nervosa (species group P) and the forb Senecio inornatus (species group M).

The total species count is 212 , which gives an average of 35.3 species per $30 \mathrm{~m}^{2}$.

\subsubsection{Cliffortia ramosissima variant}

This variant is located on the hot and dry western and northwestern aspects on moderate $\left(8^{\circ}\right)$ to steep $\left(>45^{\circ}\right)$ inclines on the summit plateau. Rock exposure is 15 to $30 \%$. Soils are shallow, from 40 to $100 \mathrm{~mm}$ deep, and well drained. No trampling was noted and there was moderate to zero soil erosion. The low sclerophylous shrub Passerina montana (species group M) covers 20 to $50 \%$ of the variant and grows at altitudes of between 2224 and $2367 \mathrm{~m}$

The dwarf shrubs Selago galpinii and Cliffortia ramosissima, along with the forb Senecio subrubrifloris (species group F), are diagnostic for this variant.

The vegetation is dominated by the sclerophyllous shrubs Cliffortia ramosissima (species group F) and Passerina montana (species group M) and the short bunch grass Digitaria monodactyla (species group $\mathrm{H}$ ). Only two species have any significance: the low shrub Selago galpinii and the forb Senecio subrubriflorus (species group F).

The total species count is 74 , which gives an average of 25 species per $30 \mathrm{~m}^{2}$.

\subsubsection{Ischyrolepis schoenoides variant}

This variant is located in full sun on the northern and eastern aspects with flat or moderate $\left(8\right.$ to $\left.16^{\circ}\right)$ slopes on the rocky summit ridge line at altitudes of 2100 to $2279 \mathrm{~m}$. Rock exposure varies between 15 and $25 \%$ and is either basalt or cave sandstone. No trampling or surface erosion was seen. Soil depths are shallow, between 40 and $180 \mathrm{~mm}$, well drained and of mixed Ib land type. Total canopy cover is 10 and $40 \%$ for the low shrubs Cliffortia ramosissima (species group F) and Passerina montana (species group $\mathrm{M}$ ) and less than $2 \mathrm{~m}$ high.

This variant has no defining species group, although it is defined by the high cover abundance of the restio Ischyrolepis schoenoides (species group G).

The vegetation is dominated by the short, leafless, grass-like restio Ischyrolepis schoenoides (species group G) and the low shrub Passerina montana (species group M). Other species of importance include the grasses Digitaria monodactyla (species group $\mathrm{H}$ ), the forb Helichrysum dasycephalum (species group $\mathrm{H}$ ), the low shrub Cliffortia nitidula, the forb Hebenstretia dura and the sedge Ficinia gracillis (species group P).

The total species count is 100, which gives an average of 25 species for $30 \mathrm{~m}^{2}$.

\subsubsection{Eragrostis capensis variant}

This variant is located on the dry northern and north-western aspects on gentle slopes ( 3 to $8^{\circ}$ ) between 2250 and $2320 \mathrm{~m}$ on the basalt summit in full sun. Rock exposure is less than $5 \%$. Soils are well drained and depths vary from medium (100 to $110 \mathrm{~mm}$ ) to (> $300 \mathrm{~mm}$ ) deep. No trampling or surface erosion was seen. The variant is low, flat grassland with limited shrub presence.

The variant is defined by species from species group $\mathrm{K}$, which includes the perennial grasses Eragrostis capensis and Pennisetum sphacelatum, plus the forbs Hypoxis costata, Oxalis depressa, Anthospermum herbaceum, Felicia filifolia, Hermannia gerrardii and Nemesia caerulea and the geophyte Ledebouria cooperi.

The vegetation is further dominated by the grasses Digitaria monodactyla (species group $\mathrm{H}$ ) and Eragrostis capensis, the forb Felicia filiformis (species group K) and the shrub Passerina montana (species group M). Species with significant cover abundance include the small sedge Bulbostylis humilis (species group I) and the forbs Hypoxis costat and Nemesia caerulea (species group K), along with the shrubs Cliffortia ramosissima (species group F) and Rhus pyroides (species group M) and the geophyte Merwilla plumbea (species group P).

The total species count is 88 , which gives an average of 29.3 species per $30 \mathrm{~m}^{2}$.

\subsubsection{Cliffortia nitidula variant}

This Cliffortia nitidula variant occurs in full sun and is scattered over all aspects on moderate $\left(0\right.$ to $\left.8^{\circ}\right)$ slopes at 2291 to $2310 \mathrm{~m}$ on the summit basalt or at 2151 to $2176 \mathrm{~m}$ on very steep $>45^{\circ}$ slopes on the scree slopes below the cliffs. Rock cover varies between 15 and $25 \%$, with very shallow, well-drained soils between 10 and $50 \mathrm{~mm}$ deep. No trampling was seen and zero erosion was evident, despite the boulder-scree terrain. Canopy cover for shrubs $<2 \mathrm{~m}$ tall is between 5 and $20 \%$. The community forms the distinct horizontal vegetation line growing halfway up the cliffs. Geologically, it is a dolerite sill that forms a narrow (5 to $10 \mathrm{~m}$ ) ledge, visible as a bench on the south side of Platberg.

This variant is defined by the succulent Crassula depressa, the sedge Cyperus rupestris, the geophyte Eucomis montana and the slender shrub Polygala virgata (species group L).

No single species dominates this community. There is significant presence of the shrubs Cliffortia nitidula (species group P) and Passerina montana (species group $\mathrm{M}$ ). The graminoid/sedge layer includes the perennial grasses Themeda triandra (species group B), Digitaria monodactyla, Helictotrichon longifolium (species group M), Eragrostis curvula and Melinis nerviglumis (species group P) and the sedges Cyperus rupestris (species group L), Carex zuluensis and Ficinia gracilis (species group P). Other species that are locally prominent are the forbs Helichrysum dasycephalum (species group $\mathrm{H}$ ) and Felicia filiformis (species group K), the succulent Crassula depressa (species group L), the low karroid shrub Chrysocoma ciliata (species group $\mathrm{M}$ ), the succulent shrub Crassula sarcocaulis (species group N) and the geophyte Agapanthus campanulatus (species group O).

The total species count is 146, which gives an average of 29.2 species per $30 \mathrm{~m}^{2}$.

2.2 Crassula sarcocaulis-Cliffortia nitidula sub-community

This sub-community covers basalt sheets and boulders on moderate slopes of between 8 and $26^{\circ}$ in full sun on the plateau 
ridge line. It is predominantly located on the north-eastern aspects at 2290 to $2315 \mathrm{~m}$. Soils are shallow (40 to $120 \mathrm{~mm}$ ), they are seasonally damp to waterlogged, the sand and gravel $<10 \mathrm{~mm}$ fine, and the soils exhibit no trampling or erosion. Rock exposure is 10 to $20 \%$.

Species belonging to species group $\mathrm{N}$ are diagnostic for this subcommunity and include the succulent shrub Crassula sarcocaulis, the geophytes Rhodohypoxis baurii, Drimia sphaerocephala and Albuca humilis and the forbs Helichrysum chionosphaerum and Senecio inaequidens.

The sub-community has no dominant species but the significant presence of the fynbos shrub Cliffortia nitidula (species group P) and the perennial grasses Aristida monticola (species group E), Digitaria monodactyla (species group $\mathrm{H}$ ) and Panicum ecklonii (species group I) is notable. Other important species include the caespitose forb Helichrysum dasycephalum, the sedge Cyperus schlecteri and the decumbent succulent Crassula depressa (species group H), the forbs Senecio rhomboideus and Agapanthus campanulatus (species group O), the fern Cheilanthus quadripinnata and the sedges Cyperus rupestrus, Ficinia gracilis and F. stolonifera (species group $\mathrm{H}$ ).

This sub-community has a high proportion of geophytes.

The total species count is 132 , which gives an average of 22 species per $30 \mathrm{~m}^{2}$.

\section{Ordination and environmental gradients}

The distribution of fynbos plant communities for Platberg along the first and third axes of the DECORANA (Hill, 1979b) ordination diagram is shown in Fig. 3. Soil moisture decreases from left to right, with soil depth reflecting this pattern along axis 1 (eigen value $=0.779$ ). The decrease in soil depth may reflect an increase in rockiness and shallow soil. The presence of pine and wattle trees on the extreme left (relevé 37265) of the ordination diagram and of well-developed grassland (sub-community 1.1, Themeda triandra) on the far right may indicate an environmental gradient of disturbance (invasion by alien species), decreasing along axis 1 from left to right. Fire is an important factor in fynbos distribution and this may be reflected by irregular occurrence on the left and more regular on the right, as seen in sub-communities 1.1, via restio, to 2.2 and Passerina montana. The environmental gradient along axis 1 may be related to exposure and frost effects and increases from left to right. Rockiness and exposure also increase as the altitude increases from left to right. This may be indicated by the physiognomy of the habitat trend on this axis.

The environmental gradient along axis 3 (eigen value $=0.498$ ) may be a combination of decreasing soil depth from the bottom to the top of axis 3 as well as of soil texture and composition, with shallow, course-grained, leached quartzitic soils at the top of the ordination diagram and with deeper, higher-organic content, finer soils at the bottom of the axis. The vegetation gradient for axis 3 from the community circled as Passerina montana through to 2.1, with the inclusion of variant 2.1.4, can be explained by the general habitat gradient.

An examination of the ordination diagram for axes 1 and 2 (eigen value $=0.580$ ) as well as for axes 2 and 3 does not clearly show related community clusters (Table 1) along clearly discernible environmental gradients. It may be that a diagonal environmental gradient exists along axes 1 and 3, from bottom left to top right, which better explains the combination of the aspect habitat and environmental gradient of physiognomy, of fire, of soil depth and composition, of exposure and frost, of soil moisture and of rockiness.

\section{DISCUSSION}

Prior to this survey, no phytosociological classification had been completed for Platberg. The description of the fynbos is part of a larger study on the entire vegetation of Platberg and has been identified as one of four distinct communities occurring on this inselberg (the others are wetlands, a woody/shrubland community and grassland). 
The sclerophyllous vegetation of the Afromontane fynbos communities of the Drakensberg region is dominated by the low shrubs Cliffortia nitidula and Passerina montana, with a less dominant but significant cover abundance of Selago galpinii and Cliffortia ramosissima (Du Preez, 1992). The geophyte Watsonia lepida, the fern Cheilanthus quadripinnata, the grasses Eragrostis curvula and Melinis nerviglumis and the two sedges Carex zuluensis and Ficinia stolonifera are common throughout. Of note is that no Protea shrubs occur on Platberg, although some Protea species have been recorded in the vicinity (the Golden Gate National Park, the Qwa Qwa National Park, the southern Drakensberg and KwaZulu-Natal as well as the northern Drakensberg).

The study resulted in the identification of 13 different fynbos vegetation units, which can be grouped into two communities, four sub-communities and seven variants.

At least 16 fynbos genera occur on Platberg, with 22 endemic or near-endemic species belonging to the Drakensberg Alpine Centre (DAC) and with 22 exotic species (alien invasives).

Fynbos on Platberg shows a species richness of 28.34 per $30 \mathrm{~m}^{2}$ (with a total 43.67 per $\mathrm{km}^{2}$ for the whole of Platberg), which is comparable with the Cape and which is moderately higher than other floristic regions in South Africa (Pond et al., 2002).

\section{Affinities with Platberg}

At plant-community level, the fynbos element on Platberg is embedded within the broader grassland biome. Numerous phytosociological and floristic studies on the grassland biome elucidate the strong floristic links that exist within several of the putative grassland bioregions (Mucina and Rutherford, 2006) and with the DAC (Bredenkamp and Brown, 2003; Grobler, Bredenkamp and Brown, 2005).

The floristic links with the fynbos on Platberg and the Cape floral kingdom are shown by the following genera, which are considered typical of the Cape centres: Cliffortia, Erica, Metalasia, Muraltia, Passerina, Pentaschistis, Restio, Schoenoxiphium and Watsonia (Bester, 1998; Cowling et al., 1992; Du Preez, 1991; Haaksma and Linder, 2002; Pond et al., 2002). All these genera, except for the Restio, are present on Platberg (Table 2) and form main communities or sub-communities (Table 1). The family Restionaceace is so dominant in the Cape floristic region that it is of great importance (Haaksma \& Linder, 2002). It is considered to be one of the diagnostic families used as a distinguishing part of fynbos. The Restionaceae genus Ischyrolepis schoenoides is found on Platberg as well as other inselbergs in the Free State (Du Preez, 1991, 1992; Du Preez and Bredenkamp, 1991; Malan, 1998) and the main Drakensberg (Carbutt and Edwards, 2004; Hilliard and Burtt, 1987; Körner, 2003; Pooley, 2003). It can be regarded as a replacement genus for the genus Restio, as its growth form is similar and phylogenetically these two genera are closely related (Haaksma \& Linder, 2002). Taxonomically, it was formally named Restio schoenoides to indicate its links with the genus Restio (Germishuizen and Meyer, 2003; Haaksma and Linder, 2002).

Two fynbos species on Platberg, Eriocephalus and Euryops, as well as the two woody/shrub species Maytenus and Diospyros are found in association with renosterveld (Pond et al., 2002) in the Western Cape. Passerina, Erica and Gnidia are also associated with fynbos (Goldblatt and Manning, 2000; Pond et al., 2002).

Proteaceae and the common genus Protea are a major component of Cape flora (Cowling et al., 1992; Goldblatt and Manning, 2000; Van Wyk and Smith, 2001). Of note is that no Protea shrubs grow on Platberg. They have, however, been recorded in the proximity at the Golden Gate National Park (Roberts, 1969) and $60 \mathrm{~km}$ to the south in Qwa Qwa (Moffett, Daemane, Pitso, Lentsoane, and Taoana, 2001), in KwaZulu-Natal, (Robbeson, 1998) and to the northern Drakensberg (Matthews, 1991) and southern Drakensberg (Bester, 1998; Hill, 1996; Smit et al., 1992). Additionally, floristic collections of Protea caffra, P. subvestita and $P$. roupelliae have been made 20 to $30 \mathrm{~km}$ to the south of Platberg (in 2003, 2004 and 2005, currently housed at the Geo Potts herbarium of the UFS). No evidence, such as excessive fire, overgrazing or the cutting of Proteas, can be found to explain this anomaly on Platberg.

\section{Affinities with the DAC}

The fynbos genera given above also occur throughout the Drakensberg (Hill, 1996; Hilliard and Burtt, 1987; Killick, 1963, 1978; Van Wyk and Smith, 2001), with Carbutt and Edwards (2004; 2006) providing the most current and complete species list and floral analysis illustrating these phytogeographic links. The fynbos species found on Platberg is, according to Acocks (1988), "different in origin and nature from the tropical vegetation" and is a vegetation type associated with poor, white, sandy soils. For Platberg, it would seem that the response to these poor soils and this habitat is the existence of the fynbos vegetation type. It is also a response to climate change over an extended period, leaving relict populations in mountainous areas (Acocks, 1988; Hilliard and Burtt, 1987; White, 1983).

\section{Fire, grazing and conservation}

Studies by Everard (1986), Seabloom and Richards (2003) and Heini, Silva and Tacheba. (2004) show that fire is a key factor influencing vegetation composition and structure, and fynbos propagation and control, with these having been extensively documented and researched (Acocks, 1988; Cowling et al., 1992; Mucina and Rutherford, 2006). The ordination for fynbos on Platberg (Fig. 3) highlights these environmental influences on the composition and structure of the sclerophyllous vegetation type.

Grazers and herbivores are important factors controlling the spread of woody vegetation (Mucina and Rutherford, 2006; Seabloom and Richards, 2003). They show that the dispersal and foraging behaviour of herbivores has the potential to structure plant communities over wide spatial and temporal ranges. On Platberg, fynbos exists on the steep sides of the Clarens formation, on rocky outcrops and on the exposed rim above the cliffs, were its location greatly reduces herbivory and trampling. More importantly, the effects of cold temperatures below freezing and high altitudes over $2200 \mathrm{~m}$ (Cowling, 1992; Mucina and Rutherford, 2006; Van Wyk and Smith, 2001) would suggest these as the main mechanism responsible for maintaining the fynbos community as well as preventing the invasion of grassland by fynbos species. by fynbos.

\section{Conservation}

The fynbos community on Platberg has moderate species richness and low alpha diversity (Table 1). However, it is embedded within the grassland biome, which has high species richness (the total floral count for Platberg is 687) (Mucina and Rutherford, 2006), although this is rapidly being fragmented, with as much as $89 \%$ transformed and between 0 and $12.53 \%$ conserved (Low and Rebelo, 1996). As a biome, it is furthermore under enormous pressure for the little remaining untransformed grassland to be developed (Bester, 1998; Low and Rebelo, 1996; Malan, 1998; Mucina and Rutherford, 2006; Perkins, Bredenkamp \& Granger, 1999).

Platberg has high terrain heterogeneity, with steep contours, deeply incised gullies and numerous slope orientations. According to Cowling et al. (1992), Low and Rebelo (1996), Mucina and Rutherford (2006), Perkins et al., (1999) and Van Wyk and Smith (2001), this provides more habitat for species and is more important than other factors in determining high biodiversity. In addition, Platberg is the single largest, bestpreserved, high-altitude grassland in the Free State (Mucina and Rutherford, 2006). 
Other, immediate threats are from the invasion of Pinus patula on the footslopes and plateau area of Platberg to the south and east. This poses a considerable conservation problem. Pinus patula is in the process of rapidly out-competing local indigenous vegetation, particularly on the cool southern slope and in the stream flowing down the gully on the south side of Platberg.

For conservation, the threat to genetic diversity (Anderson, 2001) as well as global warming are key considerations. These highlight the importance of mountains and associated inselbergs and stress the great need for and urgency of their protection. Additionally, high-altitude vegetation, specifically alpine flora as defined by White (1983) and Körner (2003), is the only biogeographic unit on land with a global distribution that accounts for nearly $3 \%$ of land surface and includes about 10000 species or about $4 \%$ of higher-order plants (Körner, 2003).

The threat posed by global warming to the world's alpine regions, including those in Africa, was predicted by Peters (1992), who indicated that as little as a $3^{\circ} \mathrm{C}$ increase in temperature for 100 years would be equivalent to a $500 \mathrm{~m}$ upward shift in altitudinal zones. For the Afromontane and Afroalpine biota this would cause a significant reduction in the distribution of plants and change their structure and composition, forcing some taxa to higher altitudes (Taylor, 1996). In some cases, such as that of Platberg, a significant reduction in taxa could also occur where the altitude is under $2500 \mathrm{~m}$.

\section{Conclusions}

Fynbos is defined as having Proteaceae, Ericaceae and Restionaceae (Goldblatt \& Manning, 2000). No Proteaceae grow on Platberg, although they have been recorded in the region. One member of the Restionaceae, namely, Ischyrolepis schoenoides, and eight members of the Ericaceae occur on Platberg. The vegetation (Table 1) shows more grass species than fynbos but, despite this, it is suggested that Platberg fynbos falls within Drakensberg-Amathole Afromontane fynbos (Gd6) vegetation as defined by Mucina and Rutherford (2006) and that it should be regarded as the species-poor Passerina montana subunit as described by Mucina and Rutherford (2006), forming an outlier of fynbos-like shrubland embedded within the broader grassland biome.

The floristic collections plus the vegetation-community analysis shows Platberg to be an important centre for biological diversity, with high species richness and a variety of habitats and complex ecosystems. It is important then that it should be given priority as a conservation site, with the development of a management plan to carry out this task.

\section{ACKNOWLEDGEMENTS}

National Geographic (grant number 7920-05) for the generous funds, without which the fieldwork and study would not have been possible.

\section{REFERENCES}

Acocks, J.P.H. 1952. Veld types of South Africa. 1st ed. Memoirs of the Botanical Survey of South Africa, 28: 1-192.

Acocks, J.P.H. 1988. Veld types of South Africa. 3rd ed. Memoirs of the Botanical Survey of South Africa, 57: 1-146.

Anderson, J.M. (ed.). 2001. Towards Gondwana alive. Pretoria: National Botanical Institute.

Barrett, A.S., Brown, L.R., Barrett, L. and Henzi, S.P. 2006 Phytosociology and plant community utilisation by vervet monkeys of the Blydeberg Conservancy, Limpopo Province. Koedoe, 49(1): 49-68.
Behr, C.M. and Bredenkamp, G.J. 1988. An analysis of the flowering plants and ferns of the Witwatersrand National Botanic Garden. South African Journal of Botany, 54(6): 515524.

Bester, S.P. 1998. Vegetation and flora of the Southern Drakensberg escarpment and adjacent areas. Unpublished MSc thesis, University of Pretoria, Pretoria.

Bredenkamp, G.J. and Brown, L.R. 2003. A reappraisal of Acocks' Bankenveld: Origin and diversity of vegetation types. South African Journal of Botany, 69(1): 7-26.

Brown, L.R., Marais, H., Henzi, S.P. and Barrett, L. 2005. Vegetation classification as the basis for baboon management in the Bourke's Luck Section of the Blyde Canyon Nature Reserve, Mpumalanga. Koedoe, 48(2): 71-92.

Carbutt, C. and Edwards, T.J. 2004. The flora of the Drakensberg Alpine Centre. Edinburgh Journal of Botany, 60(3): 581-607.

Carbutt, C. and Edwards, T.J. 2006. The endemic and nearendemic angiosperms of the Drakensberg Alpine Centre. South African Journal of Botany, 72: 105-132.

Clever, G., Brown, L.R. and Bredenkamp, G.J. 2005. The phytosociology of the Vermaaks, Markewicks and Buffelsklip valleys of the Kammanassie Nature Reserve, Western Cape. Koedoe, 48(1): 1-16.

Cowling, R.M. 1992. (ed.). The Ecology of Fynbos. Nutrients, Fire and Diversity. Cape Town: Oxford University Press.

Cowling, R.M., Holmes, P.M. and Rebelo, A.G. 1992. Plant diversity and endemism, in R.M. Cowling (ed.). The Ecology of Fynbos. Nutrients, Fire and Diversity. Cape Town: Oxford University Press.

Du Preez, P.J. 1991. A syntaxonomical and synecological study of the vegetation of the South-eastern Orange Free State and related areas with special reference to Korannaberg. Unpublished $\mathrm{PhD}$ thesis, University of the Orange Free State, Bloemfontein.

$\mathrm{Du}$ Preez, P.J. 1992. The classification of the vegetation of Korannaberg, eastern Orange Free State. I. Afromontane fynbos communities. South African Journal of Botany, 58(3): $165-172$.

Du Preez, P. J. and Bredenkamp, G.J. 1991. Vegetation classes of the southern and eastern Orange Free State, (Republic of South Africa) and the highlands of Lesotho. Navors. Nas. Mus. Bloemfontein 7: 477 - 526.

Du Toit, A.L. 1956. The geology of South Africa. Edinburgh: Oliver and Boyd.

Eckhardt, H.C.N., Van Rooyen, N. and Bredenkamp, G.J. 1993 The vegetation of the north-eastern Orange Free State, South Africa: Physical environment and plant communities of the Ea land type. Bothalia, 23(1): 117-127.

Eckhardt, H.C.N., Van Rooyen, N. and Bredenkamp, G.J. 1995. The grassland communities of the slopes and plains of the north-eastern Orange Free State. Phytocoenologia, 25(1): 1-21.

Eriksson, P.G. 1985. The depositional palaeoenvironment of the Elliot Formation in the Natal Drakensberg and NorthEastern Orange Free State. Geology Society, 88: 19-26.

Everard, D.A. 1986. The Effects of Fire on the Podocarpus latifolius Forests of the Royal Natal National Park, Natal Drakensberg. South African Journal of Botany, 52: 60-66.

Germishuizen, G. and Meyer, N.L. (eds.). 2003. Plants of southern Africa: An annotated checklist. Strelitzia, 14: 1-1231.

Goldblatt, P. and Manning, J. 2000. Cape plants. A conspectus of the Cape flora of South Africa. Strelitzia, 9: 1-743.

Grobler, C.H., Bredenkamp, G.J. and Brown, L.R. 2002. Natural woodland vegetation and plant species richness of the urban open spaces in Gauteng, South Africa. Koedoe, 45(1): 19-34.

Grobler, C.H., Bredenkamp, G.J. and Brown, L.R. 2005. Primary grassland communities of the urban open spaces in Gauteng, South Africa. South African Journal of Botany, 72: 367-377.

Haaksma, E.D. and Linder, H.P. 2000. Restios of the fynbos. Cape Town: Botanical Society of South Africa.

Heini, M., Silva, J. and Tacheba, B. 2004. Vegetation changes after a single fire-event in the Okavango Delta wetland, Botswana. South African Journal of Botany, 70(5): 695-704. 
Hennekens, S.M. 1996a. TURBOVEG. A software package for input, processing and presentation of phytosociological data. User's guide, version 2001. Wageningen: IBN-DLO.

Hennekens, S.M. 1996b. MEGATAB. A visual editor for phytosociological tables. Ulft: Giesen

Hill, M.O. 1979a. TWINSPAN: A FORTRAN program for arranging multivariate data in an ordered two-way table by classification of the individuals and attributes. New York: Cornell University, Ithaca.

Hill, M.O. 1979b. DECORANA: A FORTRAN program for detrended correspondence and analysis and reciprocal averaging. New York: Cornell University, Ithaca.

Hill, T.R. 1996. Description, classification and ordination of the dominant vegetation communities, Cathedral Peak, KwaZulu-Natal, Drakensberg. South African Journal of Botany, 62: 263-269.

Hilliard, O.M. and Burtt, B.L. 1987. The botany of Southern Natal Drakensberg. Cape Town: National Botanical Gardens.

Hoare, D.B. and Bredenkamp, G.J. 2001. Syntaxonomy and environmental gradients of the grassland of the Stormberg/ Drakensberg mountain region of the Eastern Cape, South Africa. South African Journal of Botany, 67: 595-608.

Kent, M. and Coker, P. 1992. Vegetation description and analysis: A practical approach. Chichester: J. Wiley \& Sons.

Killick, D.J.B. 1963. An account of the plant ecology of the Cathedral Peak area of the Natal Drakensberg. Memoirs of the Botanical Survey of South Africa, 34: 1-178.

Killick, D.J.B. 1978. The Afro-alpine region. In M.J.A. Werger (ed.). Biogeography and Ecology of Southern Africa. The Hague: Junk, pp. 515-560.

Körner, C. 2003. Alpine plant life. Functional plant ecology of high mountain ecosystems. Berlin: Springer-Verlag.

Land Type Survey Staff. 1991. Land types of the map 2828 Harrismith. Pretoria: Department of Agriculture.

Loock, J.C., Praekelt, H.E., Van der Westhuizen, W.A. and Welman, J. 1991. The Dewetsdorp-Thaba Nchu Mountain excursion. Bloemfontein: National Museum.

Low, A.B. and Rebelo, A.G. (eds.). 1996. Vegetation of South Africa, Lesotho and Swaziland. Pretoria: Department of Environmental Affairs and Tourism.

Macarthur, R.H. and Wilson, E.O. 2001. The theory of island biogeography. Princeton: Princeton University Press.

Malan, P.W. 1998. Vegetation ecology of the Southern Free State. Unpublished $\mathrm{PhD}$ thesis, University of the Orange Free State, Bloemfontein.

Matthews, W.S. 1991. Phytosociology of the North-eastern Mountain Sourveld. Unpublished MSc Thesis, University of Pretoria, Pretoria.

Moffett, R.O., Daemane, M.E. Pitso, T.R., Lentsoane, R. and Taoana, T.R.N. 2001. A Checklist of the Vascular Plants of Qwa-Qwa and notes on the Flora and Vegetation of the Area. UNIOWA Research Chronicles, 3(2): 32-83.

Moon, B.P. and Darids, G.F. (eds.). 1992. The geomorphology of Southern Africa. Halfway House: Southern Book Publishers.

Mucina, L. and Rutherford, M.C. 2006. The vegetation of South Africa, Lesotho and Swaziland. Strelitzia, 19: 1-807.

Mueller-Dombois, D. \& Ellenberg, H. 1974. Aims and methods of vegetation ecology. New York: J. Wiley \& Sons.

Mutke, J., Kier, G., Braun, G., Schultz, C.H.R. and Barhlott, W. 2001. Patterns of African vascular plant diversity: A GISbased analysis. Syst. Geogr. Pl., 71: 1125-1136.

Parmentier, I., Oumorou, M., Porembski, S., Lejoly, J. and Decocq, G. 2006. Ecology, distribution and classification of xeric monocotyledonous mats on inselbergs in West Africa and Atlantic central Africa. Phytocoenology, 36(4): 547-564.

Perkins, L., Bredenkamp, G.J. and Granger, J.E. 1999. The phytosociology of the incised river valleys and dry upland savannah of the southern KwaZulu-Natal. South African Journal of Botany, 65(5/6): 321-330.

Peters, R.L. 1992. Conservation of biological diversity in the face of climatic change. In R.L. Peters, \& T.E. Lovejoy (eds.) Global Warming and Biological Diversity. New Haven: Yale University Press, pp. 15-30.

Pond, U., Beesley, B.B., Brown, L.R. and Bezuidenhout, H. 2002. Floristic analysis of the Mountain Zebra National Park, Eastern Cape. Koedoe, 45(1): 35-58.

Pooley, E. 2003. Mountain Flowers. A field guide to he Flora of the Drakensberg and Lesotho. The Flora Publishing Trust, Durban.

Porembski, S. and Brown, G. 1995. The vegetation of inselbergs in the Comoé National Park (Ivory Park). Conservatoire et Jardin Botanicues de Geneve, 50(2): 351-365.

Robbeson, R.A.J. 1998. Phytosociology of northwestern KwaZuluNatal. MSc thesis, University of Pretoria, Pretoria.

Roberts, B.R. 1969. The vegetation of the Golden Gate Highlands National Park. Koedoe, 12: 15-28.

Sarthou, C. and Villiers, J-F. 1998. Epilithic plant communities on inselbergs in French Guiana. Journal of Vegetation Science, 9: 847-860.

Schulze, R.E. 1997. South African atlas of agro hydrology and climatology. Pretoria: Water Research Commission.

Scott, L. 1988. Late quaternary vegetation history and climatic change in the eastern Orange Free State, South Africa. South African Journal of Botany, 55(1): 107-116.

Seabloom, E.W. and Richards, S.A. 2003. Multiple Stable Equilibrium in Grassland mediated by Herbivore Population Dynamics and Foraging Behaviour. Ecology, 84(11): 2891-2904.

Segal, S. 1969. Ecological notes on wall vegetation. PhD thesis, University of Amsterdam, Amsterdam.

Soil classification working group, 1991. Soil classification. A taxonomic system for South Africa. Memoirs on the agricultural natural resources of South Africa. No 15. Dept. of Agricultural Development, Pretoria, pp. 1-262.

Smit, C.M., Bredenkamp, G.J. and Van Rooyen, N. 1992. Phytosociology of the Ac land type in the foothills of the low Drakensberg in north-western Natal. South African Journal of Botany, 59: 203-214.

Smit, C.M., Bredenkamp, G.J. and Van Rooyen, N. 1995. The grassland vegetation of the low Drakensberg escarpment in the north-western KwaZulu-Natal and north-eastern Orange Free State border area. South African Journal of Botany, 61: 9-17.

South Africa Weather Services. 2007. Climate of South Africa. Climate statistics for Bethlehem from 1961-1990. WB 40. Pretoria: Government Printer.

Taylor, D. 1996. Mountains. In: W.M. Adams, A.S. Goudie \& A.R. Orme (eds.) The Physical Geography of Africa. Oxford: Oxford University Press, pp. 287-306.

Truswell, J.F. 1970. An introduction to the historical geology of South Africa. London: Purnell Press.

Van Wyk, A.E. and Smith, G.F. 2001. Regions of floristic endemism in Southern Africa: A review with emphasis on succulents. Pretoria: Umdaus Press.

Van Zinderen Bakker Jr, E.M. 1973. Ecological investigation of forest communities in the Eastern Orange Free State and the adjacent Natal Drakensberg. Vegetatio, 28: 299-334.

Westhoff, V. and Van der Maarel, E. 1980. The Braun-Blanquet approach. In: R.H. Whitaker, (ed.) Classification of Plant Communities. The Hague. Junk: Kluwer Academic Publisher, pp. 287-378.

Whittaker, R.H. (ed.). 1980. Classification of plant communities. The Hague: Junk: Kluwer Academic Publisher.

White, F. 1983. The vegetation of Africa. Volume XX, a descriptive memoir to accompany the Unesco/AETFAT/UNSO vegetation map of Africa. Paris: Unesco. 